\title{
PROTOCOL: Mixed-methods systematic review to determine the effectiveness of museums using virtual field trips for STEM education
}

\author{
Moïse-Denis K. Jean ${ }^{1} \&$ C. Aaron Price ${ }^{1}$ \\ ${ }^{1}$ Department of Research and Evaluation, Museum of Science and Industry, Chicago
}

\begin{abstract}
Due to the COVID19 pandemic, many formal and informal educational institutions, such as schools and museums, have closed or else been forced to alter their pedagogical techniques. To keep students actively engaged in specific topics, museums are incorporating virtual field trips (VFTs) for schools to utilize. The goal of this review is to determine what impact VFTs have on school-aged audiences, to recognize the factors that contribute to successful VFTs, and to evaluate evidence that VFTs can assist all communities and schools that do not have access to informal education. This protocol articulates the methodological steps the researchers use.
\end{abstract}




\section{Background}

\subsection{Museum field trips as informal education}

Informal educational settings, such as museums or afterschool clubs, are alternative methods for students to learn a wide range of subjects through social interaction and participation (Ramey-Gassert, 1994). School field trips to museums have been found to develop students' creativity and expertise in different subject areas and fields, such as art (Mahgoub, 2014), and science (Feinstein, 2014). Students may also teach each other and take part in discussions with their classmates. These experiences enhance student engagement and strengthen students' understanding of different scientific concepts (Behrendt, 2014). Science museums are essential to delivering valuable information to the public, especially if the audience has a clear motive, learning objective, or prior knowledge on the science topic before attending the museum (Falk, 1986). Successful field trips include planned experiences, activities for the students, teacher-student participation, trained chaperones, and they provide a direct connection with the curriculum (Behrendt, 2014). The facilitation of student interest and participation in learning through museum field trips can be one of the most beneficial strategies for student growth.

Though these types of educational settings provide benefits to young student learners, such as better understanding of the topic, stronger class community, and a heightened relationship with instructional staff (Leydon, 2013), barriers exist that prevent some student groups from experiencing them. Depriving these student groups from accessing this type of educational service is not only a disservice to these communities, but also an injustice to those most in need. Children from a low social-economic status are less likely to visit informal education institutions than children from a higher social-economic status (Swan, 2014; DeWitt, 2017). Barriers of access vary from lack of transportation, safety, restrictions of time, large class size, conflicts of schedule, inflexibility of curriculum, and lack of venue options (Michie, 1998; Elleven, 2006; Leydon, 2013). Some of these issues can be alleviated using virtual field trips (VFTs).

\subsection{Advantages of virtual field trips}

What makes for a good virtual field trip (VFT)? Many of the same criteria for successful inperson field trips also apply for VFTs. This includes pre- and post-lesson support and active teacher participation (Kenna, 2018). As technology improves, the need to incorporate advancements, such as games or communication methods, into educational frameworks increases. Teachers have increasingly implemented computer games into their lesson plans to better explain concepts to their classes, such as using Angry Birds to teach physics principles or Minecraft to teach geometry problems dealing with volume (Bender, 2017). Students with disabilities might have difficulties with inclusion among their classmates during in-person field trips, and VFTs might help since they have been found to positively affect career development among students with disabilities (Elleven, 2006). 
Evidence has shown that technological advances in education are incredibly beneficial. A meta-analysis has concluded that virtual reality teaching methods have an incredibly positive impact on learning among undergraduate students compared to those unexposed (Hillstrom, 2019). Some of the key elements of successful virtual reality teaching methods identified were moderately immersive virtual reality (such as a 3D desktop system), and place-based experiences. VFTs can be used as support for future in-person field trips (CoxPeterson \& Melber, 2001) since VFTs are designed to replicate them. VFTs can be more beneficial than in-person since some formats let teachers adapt their lessons and provide supplemental materials (Kirchen, 2011).

A study expands on using VFTs to boost the in-person field trip experience for university students. This study used both qualitative and quantitative methods to evaluate the significance of VFTs focusing on engineering. The students saw that in-person field trips allowed them to see the application of theoretical concepts and speak with experts in the field. But without using VFTs before, the effectiveness of the in-person field trips declined substantially (Seifan, 2019). This displays one of the advantages of VFTs.

\subsection{Effects of field trips during a global pandemic}

The coronavirus (COVID-19) pandemic is one of the greatest acute educational challenges of recent years. This highly contagious infectious respiratory disease caused additional issues beyond health. To prevent the incidence rate, closures from multiple institutions occurred, such as theatres, museums, sporting events (Király, 2020). In a typical year, the Museum of Science and Industry, Chicago (MSI Chicago) hosts about 300,000 children who visit as part of a school field trips. All those students visit for free and bus transportation is provided for lower-resourced schools. For example, in 2014-2015, 61\% of the visiting to participate in Learning Labs sessions were students categorized as "lower" or "lower-middle" class by their teachers. In the 2020-2021 academic year, all field trips to the museum were suspended meaning these students did not get the same in-person experience. As with many museums, MSI Chicago rolled out virtual versions of some of their field trip programming. However, it was a challenge as the museum's expertise is in hands-on, inperson, high-inquiry learning and engagement. How to pivot to such a different world in such a brief time was an issue many museums faced. This shows how crucial of professional development among informal science educators is regardless of the topic or skill needed (Ennes, 2020).

\subsection{Intervention}

The evolution of technology makes it difficult to compare different methods of VFTs when organizing a systematic review. The Bender definition of virtual field trips is "the use of distance education technologies (Internet, virtual reality, television, satellite, or recorded videos) to help students travel to and study a specific location of interest while physically remaining in their classroom (2017)." 
Though this is a definition of VFTs, this systematic review will focus on more advanced options that include interactions between the media platform and audience. VFTs have their own challenges, such as an additional workload on the teachers or a lack of access to physical resources like lab materials or exhibit technology. Visitors have found that though virtual content has motivated them to different activities and exhibits, it is more socially and physically isolating and less appealing when not well-organized with exhibits on the museum floor (Hsi, 2003). This explains the need for the field to know how to offer staff members professional development to provide the optimal experience for all audiences, especially young students. To narrow the large audience, this paper will focus on VFTs about a STEM topic among middle and high school students (between the ages of 12-18). This will then create a time restriction for studies and sources due to the drastic technological changes throughout the years. Only literature from the past two decades will be included (between 2000-2020).

\subsection{How intervention might work}

Quality VFTs have been known to have specific components to enhance educational outcomes. This systematic review will adopt a VFT logic model by Sriarunrasmee et al. (2015) that develops science learning outcomes among eighth grade students (Figure 1). This model articulates a formulaic approach of the learning process and engagement skills within a VFT. The model consists of five components of a VFT and three phases of learning activities, which consisted of six learning steps.

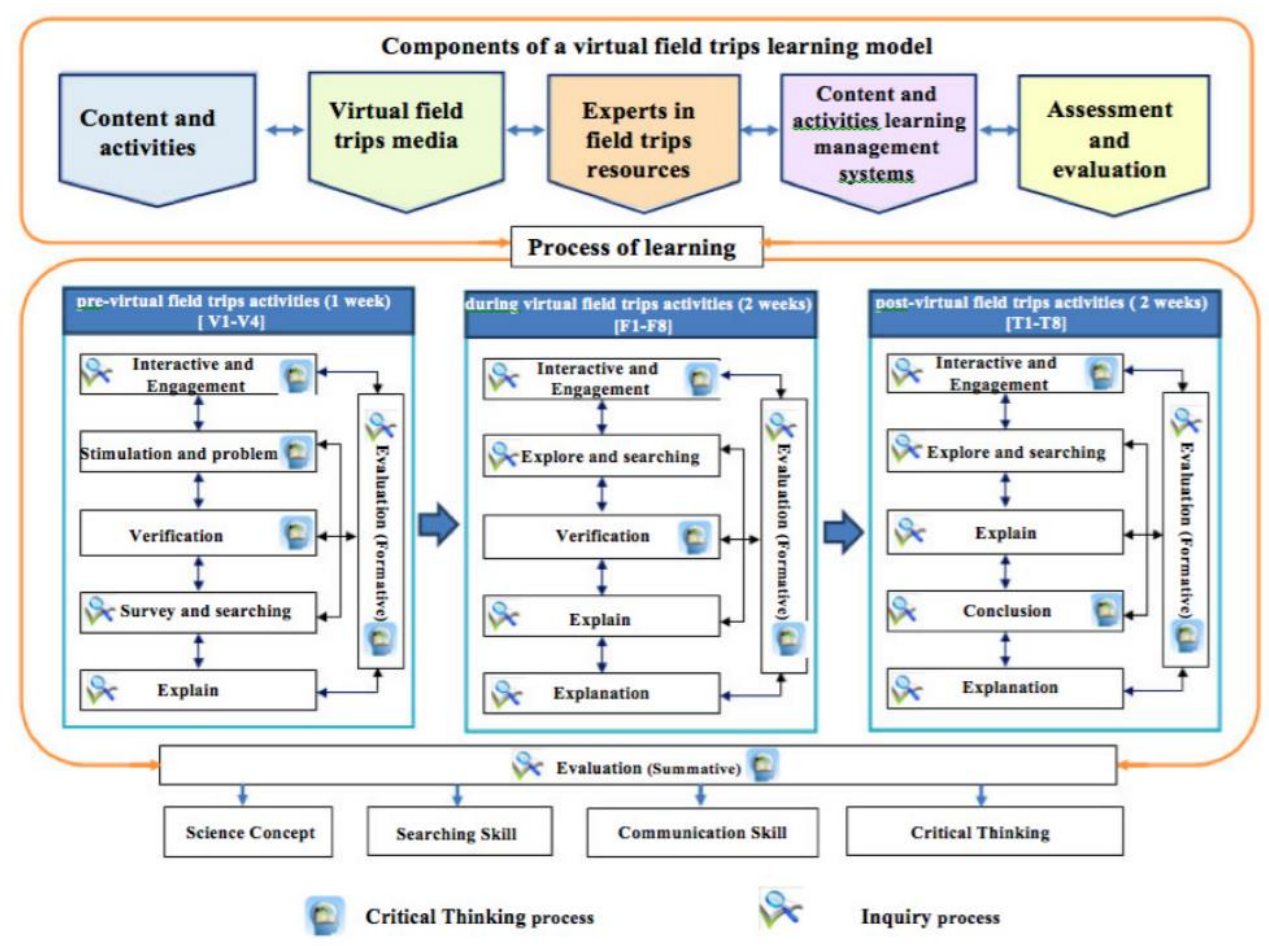

Figure 1. Virtual field trip logic model with inquiry learning and critical thinking to enhance the science learning outcome of lower secondary students from Sriarunrasmee et al. (2015). 
This model was ideal for this systematic review because it synthesizes information from literature on VFTs to design the different components and steps used and it focuses on same population of interest (secondary education students) as this review. The five components of the learning model are: 1) content and activities; 2) virtual field trips medias including video clips, pictures, animations, online diaries, worksheets, activity sheets, and games; 3) experts in field trips recourses; 4) learning management system for victual field trips; and 5) assessment and evaluation. Because some literature will use in-person field trips and might use VFTs as a pre-or-post activity, the three learning activities for this review will be "pre-field trip activities," "during field trip activities," and "post-field trip activities." All the six steps within the learning activities will remain the same: engagement, investigation and exploration, explanation, conclusion, elaboration, and evaluation (formative). This review will view the qualitative information within the collected literature for the evaluation (summative) portion of the model to discern the four skills the students developed (science concepts, searching skill, communication skill, and critical thinking).

\subsection{Why it is important to do the review}

The information of any subject learned in a lecture is crucial for students to apply it into real world settings (Biggs, 2011). Viewing the taught information through a field trip format helps students recognize how these concepts are used in practice. Since the average student attention span begins to decline after 15 minutes, long traditional lectures have a major effect on student engagement and learning (Bradbury, 2016). Lectures are designed to be developed and enhanced with different ideas and techniques (Bligh, 1999), and field trips can diversify the pedagogy.

Researchers predict that this mixed-method systematic review will help museums better integrate VFTs into their education plans to reach audiences who cannot visit museums. The authors assume that there is not much substantial quantitative research on this topic, but by also analyzing qualitative and mixed-methods research papers, the validity of the findings will be augmented by data triangulation. If learning from and engagement with VFTs is aligned with the subjects taught in school, many teachers can implement this into their lesson plans. These field trips might be more beneficial for some educators to use in their classes to advance the students' comprehension after an in-person field trip.

While VFTs should not replace in-person field trips, they can prepare most student for an inperson field trip or enhance what was learned from a previous in-person field trip visit (Spicer, 2001). This study will allow museums to understand what different educational strategies are beneficial for students about informal education from museums. Ultimately, this new information may lead to more students accessing these different resources. A stronger understanding of VFTs and educational strategies for museums to utilize might develop the breadth of students accessing these resources. By collecting both qualitative and quantitative information, this systematic review is planned to have a more advanced solution in regard to how science museums use VFTs. 


\section{Objectives}

This mixed-method systematic review is an extension from a National Science Foundation experimental study at the Museum of Science and Industry at Chicago. It focuses on examining how a structured field trip experience using real medical training materials may impact STEM learning and career interest among students and how that impact may be related to racial and ethnic identity. As part of the study, some students will experience a VFT instead of or in addition to an in-person field trip.

Because of the COVID-19 pandemic, the study was suspended, but the researchers recognized the importance of exploring the effectiveness of VFTs. This systematic review specific objectives are:

1. To systematically identify what impact VFTs have on school-aged audiences.

2. To recognize the factors that contribute to successful VFTs.

3. To evaluate evidence that VFTs can assist all communities and schools that do not have access to informal education.

\subsection{Research questions}

1. Is there a difference in STEM science learning outcomes between VFTs and traditional education among students $12-18$ years old?

2. What are the effects of VFTs on students 12-18 years old in STEM education?

3. What are the best practices for designing STEM-focused, museum-based VFTs or how do museums make VFTs better?

4. What are the differences between the impact of VFTs among those with different demographics and cultural backgrounds?

\section{Methodology}

The Preferred Reporting Items for Systematic review and Meta-Analysis Protocols (PRISMA-P) was used to prepare this systematic review protocol because of its overall guidance to structure and design a systematic review and meta-analysis (Shamseer, 2015). PRISMA-P is used for researchers to a complete an accurate methodological approach when conducting a systematic review or meta-analysis. This provides readers with transparency and an opportunity to view the concise decision making from the researchers. This review will include studies published in peerreview journals, articles published from scientific museum websites, expertise from professionals in the field of museum education, among other sources that discuss VFTs and STEM.

\subsection{Inclusion and exclusion criteria of studies for review}


Informal learning institutions such as museums often are not associated with colleges, government or other institutions that have access to libraries and budgets for peer-review journals. As a result, much of their scholarship exists in the form of grey literature. These include white papers, technical reports and other forms of publication that have a peer review aspect but not necessarily the same peer review rigor as traditional research journals (Benzies, 2006). Thus, for this review we will include studies irrespective of their publication status (i.e empirical studies in peer-review journals, non-published, or on-going). Two existing search criteria will be used in practice to determine whether articles appropriately fit for review in this paper. The PICOS (Population, Intervention, Comparison, Outcome, Study Design) method will be used to determine conceptual and operational definitions that are relevant to the research from quantitative articles, while the SPIDER (Sample, Phenomenon of Interest, Design, Evaluation, Research type) method will be used for qualitative and mixed-methods research (Methley, 2014) (Table 1).

\begin{tabular}{|c|c|c|c|}
\hline \multicolumn{4}{|c|}{ Inclusion Criteria } \\
\hline \multicolumn{2}{|r|}{ Quantitative } & \multicolumn{2}{|c|}{ Qualitative and Mixed Methods } \\
\hline Population & $\begin{array}{l}\text { Students between the ages of } \\
12-18\end{array}$ & Sample & $\begin{array}{l}\text { Students between the ages of } \\
12-18\end{array}$ \\
\hline Intervention & $\begin{array}{l}\text { Virtual field trips on STEM } \\
\text { education }\end{array}$ & $\begin{array}{l}\text { Phenomenon of } \\
\text { Interest }\end{array}$ & $\begin{array}{l}\text { Important elements in creating } \\
\text { virtual field trips to enhance } \\
\text { STEM education }\end{array}$ \\
\hline $\begin{array}{l}\text { Comparison } \\
\text { (optional) }\end{array}$ & $\begin{array}{l}\text { Formal education or in-person } \\
\text { field trips }\end{array}$ & Design & $\begin{array}{l}\text { Literature of any research } \\
\text { design, grey literature, } \\
\text { interviews with experts }\end{array}$ \\
\hline Outcome & $\begin{array}{l}\text { STEM learning and student } \\
\text { engagement }\end{array}$ & Evaluation & $\begin{array}{l}\text { Point of view of students, } \\
\text { educators, and designers on } \\
\text { efficient virtual field trips }\end{array}$ \\
\hline Study design & $\begin{array}{l}\text { Experimental, quasi- } \\
\text { experimental }\end{array}$ & Research type & Qualitative or Mixed Methods \\
\hline
\end{tabular}

Table 1. Inclusion criteria using the PICOS and SPIDER search tools

Inclusion:

- VFTs on STEM education

- Articles including students between the ages of 12-18

- Studies published from the year 2000 and later

- Interviewers or transcripts regarding VFT usage

- Peer review published, pre-published, or on-going research

\section{Exclusion:}

- Papers published before 2000

- Using antiquated forms of VFTs (i.e. television or recorded video)

- Papers not translated into English

- Papers that do not have data (reviews, position papers, etc.) 


\subsection{Types of study designs and research type:}

This systematic review will comprise of empirical studies in peer-review journals, non-published, or on-going that use quantitative, qualitative or mixed methods research. The reviewers do not anticipate many randomized experimental studies to be in the literature to explore true causation between STEM learning and VFTs. All qualitative and mixed methods studies will be incorporated for an explanatory analysis of the results from the quantitative analysis. Interviews will also be conducted to complete any gaps that are present within the qualitative portion of the review.

\subsection{Types of sample/population:}

The reviewers will include studies focusing on students between the ages of 12-18 who are taking part on STEM education. If we do not initially find enough qualifying articles to do an analysis, we may expand to include studies of VFTs aimed at college students. Specific communities are often excluded from informal education programs, such as students in lowincome communities or students with disabilities. To capture the effectiveness of VFTs on a larger range of students, research studies focusing on disadvantaged groups will be specifically searched.

\subsection{Types of interventions/phenomena of interest:}

The included studies will involve VFTs centered around STEM topics. The phenomenon of interest will be ideas and suggestions from students, educators, and designers to hone VFTs in the museum setting. Furthermore, these interviews or studies might include portions about how VFTs are salient for specific demographic groups.

\subsection{Comparison group:}

A comparison group will be an optional requirement for the studies included in this review because the reviewers will be looking at studies with various research designs. The studies that do have a comparison group would most preferably compare VFTs to different educational methods, such as in-person field trips or traditional formal education settings.

\subsection{Types of outcome measures/evaluation:}

For this study, both STEM-VFT effectiveness (assessed by quantitative studies) and efficiency (assessed through qualitative studies) are the measures of interest. This review hopes to increase the literature on the importance for museums to utilize VFTs to expand their impact beyond museum-attending guests. The review will also explore how and why these VFTs did or did not work to help inform future designs of VFTs.

\subsection{Types of settings:}


Primarily, museums and other informal education settings are the primary settings of interest. But because of the limited research on this topic, sources using VFTs within the formal education settings will be included. The topic of the course and VFT will encompass STEM (science, technology, engineering, or math) education.

\section{Search strategies for identification of studies and sources}

The first author will develop an articulate search strategy to identify articles and sources associated with the review. For example, titles and abstracts will be screened based on type of VFT, phenomenon of interest, study design, and participants. A diagram of the screening process on Figure 2 follows the PRISMA study selection flowchart. A second reviewer will independently screen the selected studies, as well. Studies will be searched from 2010 onward and must be available in English. The screening for this review will continue through a full-text screening by the same reviewers. If any selection of sources were agreed upon by the two reviewers, a third will discuss these differences to complete the selections.

All published and non-published studies will be searched through common databases. Table 2 is an example that outlines the ERIC search strategy for terms. Once all the sources are screened and found, the reviewers will contact the authors of the articles for further information, if needed. Also, more information will be collected after meetings with the education coordinators at the Museum of Science and Industry - Chicago about non-published or current studies or other work regarding VFTs. These individuals are experts in the field of STEM education at the museum. Below are where the reviewer will collect the information from specific formats. The following search keywords will be used:

\section{Electronic databases:}

- Google Scholar

- ERIC

- PubMed

Grey literature searches and websites:

- Greylit.org

Handsearching and snowballing techniques:

Included articles will also be hand-searched to decrease the likelihood that relevant literature would be missed. The reviewers will use both backward and forward snowball searching to check more literature quicker and efficiently.

\section{Contacting experts:}

Experts from the Museum of Science and Industry - Chicago Student Experiences department will be consulted to provide their advice and lead the reviewers to other sources of current or non-published work in the field. 


\begin{tabular}{|ll|}
\hline Search & Search Terms \\
\hline S1 & Title:"virtual field trip" \\
S2 & Abstract:"virtual field trip" \\
S3 & S3 since 2012 \\
\hline S4 & $\begin{array}{l}\text { Abstract:virtual field trip } \\
\text { experiment }\end{array}$ \\
\hline S5 & Abstract:virtual field trip STEM \\
\hline S6 & Abstract:virtual field trip qualitative \\
S7 & Virtual reality STEM education \\
\hline
\end{tabular}

Table 2. Search strategy in ERIC 


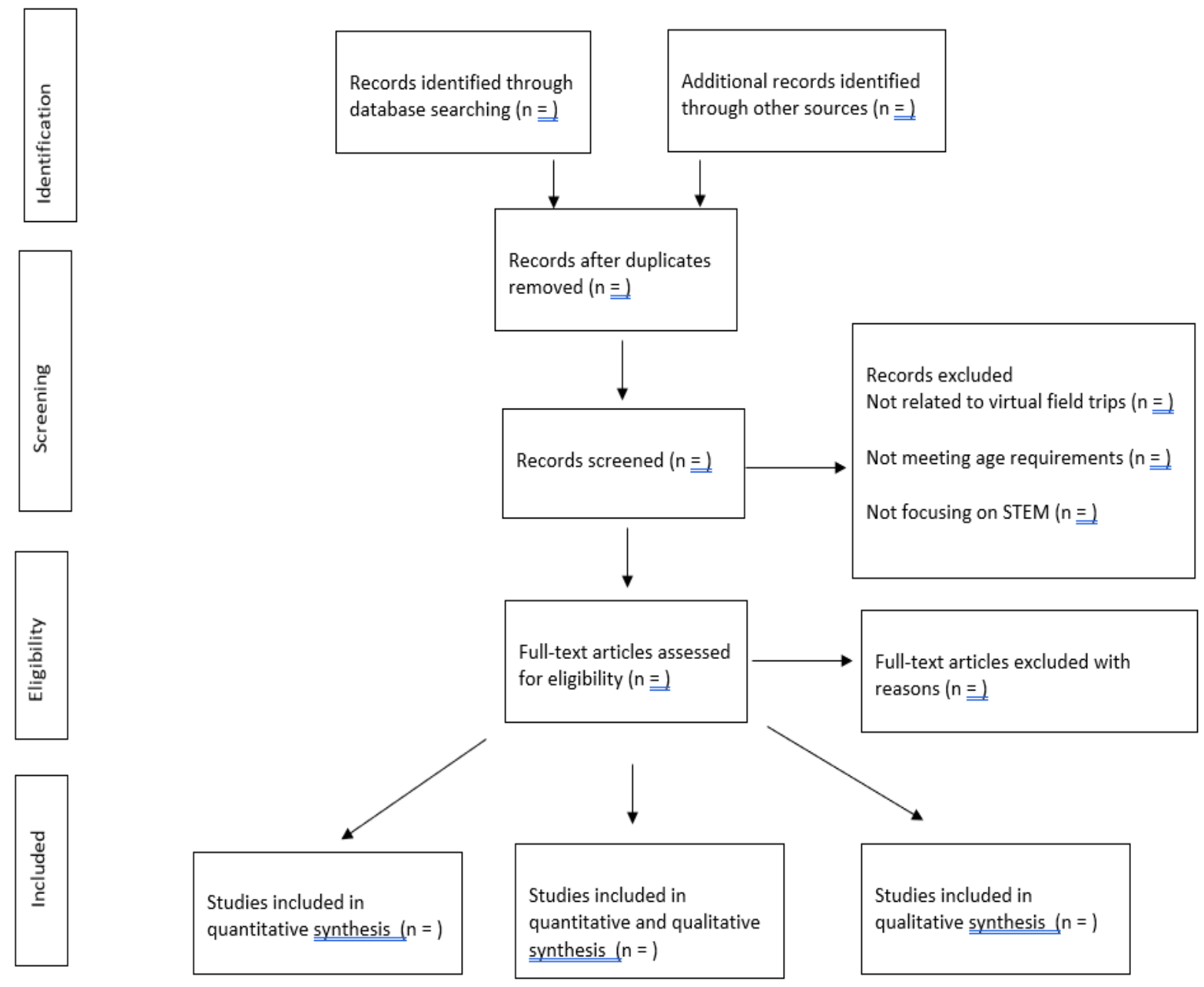

Figure 2. PRISMA flowchart of screening process of virtual field trip sources for quantitative, qualitative and mixedmethods research

\section{Risk of bias and quality assessment}

The risk of bias and methodological quality of the sources included in this review will be assessed by the reviewers using the Mixed Methods Appraisal Tool (MMAT) (Pluye, 2011). This assessment tool appraises of five types of research studies: qualitative research, randomized controlled trials, non-randomized studies, quantitative descriptive studies, and mixed methods studies. Two reviewers will use this tool independently, and use a third reviewer during a discussion if their assessments of the study quality are at a disagreement.

\section{Data extraction, coding, and management}

A coding framework is developed as a strategy to categorize these studies. Table 3 displays the coding process. Two reviewers will use this coding frame and a third reviewer will be introduced in case of coding disagreement by the first two reviewers. The software Review Manager version 5.4 will download the titles and abstracts from the electronic databases. 


\begin{tabular}{|ll}
\hline Category & Sub-categories \\
\hline Publication status & A. Completed \\
\hline Study design & B. On-going \\
& A. Quantitative \\
\hline Comparison group & B. Qualitative \\
& C. Mixed Methods \\
\hline Type of comparison group & A. Yes \\
& B. No \\
\hline Outcome & A. In-person field trip \\
& B. Formal education \\
\hline Setting & C. N/A \\
\hline Type of intervention & D. Student Engagement \\
& E. STEM knowledge \\
& F. Opinion/Point of view \\
\hline
\end{tabular}

Table 3. Coding framework

Abbreviations:

1. MMAT: Mixed Methods Appraisal Tool

2. MSI: Museum of Science and Industry - Chicago

3. PICOS: Population, Intervention, Comparison, Outcome, Study Design

4. PRISMA-P: Preferred Reporting Items for Systematic review and Meta-Analysis Protocols

5. SPIDER: Sample, Phenomenon of Interest, Design, Evaluation, Research type

6. STEM: Science technology engineering and mathematics

7. VFTs: Virtual Field Trips 


\section{References}

American Alliance of Museums. (2020). National Survey of COVID-19 Impact on United States Museums. https://www.aam-us.org/wp-content/uploads/2020/07/2020_National-Survey-of-COVID19-Impact-onUS-Museums.pdf

Behrendt, M. \& Franklin, T. (2014). A review of research on school field trips and their value in education. International Journal of Environmental \& Science Education, 9, 235-245.

Bender, W.N. (2017). 20 Strategies for increasing student engagement. Learning Sciences International. Benzies, K.M. Premji, S., Hayden, K.A., \& Serrett, K. (2006). State-of-the-evidence reviews: Advantages and challenges of including grey literature. Worldviews on Evidence-Based Nursing, 3(2): 55-61.

Biggs, J.B. \& Tang, C. (2011). Teaching for quality learning at university ( $4^{\text {th }}$ ed.). Open University Press.

Billock, J. (September 16, 2020). How will Covid-19 change the way museums are built.

Smithsonianmag.com https://www.smithsonianmag.com/travel/how-will-covid-19-change-way-futuremuseums-are-built-180975022/

Bligh, D.A. (1998). What's the use of lectures? Intellectual Books: England, UK.

Bradbury, N.A. (December 01, 2016). Attention span during lectures: 8 seconds, 10 minutes, or more? Advances in Physiology Education. 40(4): 509-13. doi: 10.1152/advan.00109.2016.

Cox-Petersen, A. M., \& Melber, L. M. (2001). Using technology to prepare and extend field trips. The Clearing House, 75(1), 18-20.

DeWitt, J. \& Archer, L. (2017). Participation in informal science learning experiences: the rich get richer? International Journal of Science Education, 7(4): 356-73.

Elleven, R., Wircenski, M., Wircenski, J., \& Nimon, K. (2006). Curriculum-based virtual field trips: Career development opportunities for students with disabilities. Journal for Vocational Special Needs Education, 28(3), 4-11.

Ennes, M., Jones, M.G., \& Chesnutt, K. (August 27, 2020). Evaluation of educator self-efficacy in informal science centers. Journal of Museum Education, 45(3): 327-39.

Falk, J.H., Koran, J.J., \& Dierking, L.D. (1986). The things of science: Assessing the learning potential of science museums. Science Education, 70(5): 503-8.

Feinstein, N.W., \& Meshoulam, D. (2014). Science for what public? Addressing equity American science museums and science centers. Journal of Research in Science Teaching, 51(3), 368 - 394.

Hillstrom J.E. (2019). Virtual Place-Based Learning in Interdisciplinary Contexts: A Psychological Perspective and a Meta-analytic Review. In: Lansiquot R., MacDonald S. (eds) Interdisciplinary Perspectives on Virtual Place-Based Learning. Palgrave Pivot, Cham. http://doi-org443.webvpn.fjmu.edu.cn/10.1007/978-3-030-32471-1 2.

Hsi, S. (2003). A study of user experiences mediated by nomadic web content in a museum. Journal of Computer Assisted Learning, 19, 308 - 19. 
Kendall, J. J. (2021). Invisible Doors The Hybrid Museum: Early Childhood Virtual \& In-Person Learning in Art Museums (Doctoral dissertation).

Kenna, J.L. \& Potter, S. (2018). Experiencing the world from inside the classroom: Using virtual field trips to enhance social studies instruction. The Social Studies, 109(5): 265-275.

Király, O. et al. (July 2020). Preventing problematic internet use during the COVID-19 pandemic: Consensus guidance. Comprehensive Psychiatry, 100.

Kirchen, D. J. (2011). Making and taking virtual field trips in pre-k and the primary grades. YC: Young Children, 66 (6), 22-26.

Lantz, E. (2011). Planetarium of the future. Curator: The Museum Journal, 54(3): 293-312.

Leydon, J. \& Turner, S. (2013). The challenges and rewards of introducing field trips into a large introductory geography class. Journal of Geography, 112(6): 248-61.

Mahgoub, Y.M. \& Alawad, A.A. (January 2014). The impact of field trips on students' creative thinking and practices in art education. American Journal of Science. 10(1): 46-50.

Methley AM, Campbell S, Chew-Graham C, et al. PICO, PICOS and SPIDER. a comparison study of specificity and sensitivity in three search tools for qualitative systematic reviews. BMC Health Serv Res 2014;14:579.doi:10.1186/s12913-014-0579-0pmid:http://www.ncbi.nlm.nih.gov/pubmed/2541315

Michie, M. (1998). Factors influencing secondary science teachers to organize and conduct field trips. Australian Science Teacher's Journal, 44, 43-50.

Pluye P, Robert E, Cargo M, Bartlett G, O'Cathain A, Griffiths F, Boardman F, Gagnon MP, Rousseau MC (2011). Proposal: a mixed methods appraisal tool for systematic mixed studies reviews.

[http://mixedmethodsappraisaltoolpublic.pbworks.com].

Seifan, M., Dada, D., \& Berenjian, A. (2019). The effects of virtual field trip as an introductory tool for an engineering real field trip. Education for Chemical Engineers, 27: 6-11.

Shamseer L, Moher D, Clarke M, Ghersi D, Liberati A, Petticrew M, Shekelle P, Stewart L, PRISMA-P Group. Preferred reporting items for systematic review and meta-analysis protocols (PRISMA-P) 2015: elaboration and explanation. BMJ. 2015 Jan 2;349(jan02 1):g7647.

Spicer, J.I. \& Stratford, J. (December 20, 2001). Student perceptions of a virtual field trip to replace a real field trip. Journal of Computer Assisted Learning, https://doi.org/10.1046/j.0266-4909.2001.00191.x.

Swan, D.W. (2014). Children who visit museums have higher achievement in reading, math, and science. Institute of Museum and Library Services. Retrieved from https://www.imls.gov/newsevents/upnext-blog/2014/04/children-who-visit-museumshave-higher-achievement-reading-math-and

Ramey-Gassert, L., Walberg, H.J., III, \& Walberg, H. J. (1994). Re-examining connections: Museums as science learning environments. Science Education, 78, 345-363. 